\title{
Improving Detection Rates of Giardia Using Duodenal Biopsy PCR: Is the Juice Worth the Squeeze?
}

\author{
Jeffrey A. Berinstein ${ }^{1} \cdot$ Krishna Rao ${ }^{2}$ \\ Published online: 26 February 2020 \\ (c) Springer Science+Business Media, LLC, part of Springer Nature 2020
}

Acute diarrhea is one of the most common illnesses worldwide, estimated to be the ninth leading cause of death globally according to the Global Burden of Disease Study [1]. Worldwide, infectious pathogens account for the majority of cases of diarrhea, especially among low-income populations with limited access to safe water, sanitation, and urgent medical care, though infectious etiologies of diarrhea remain an important issue even among resource-rich populations.

Giardia duodenalis (also known as G. lamblia or G. intestinalis), a protozoan parasite, is common among resourcelimited countries with an estimated prevalence of $20-40 \%$ [2]. Even in resource-rich countries, Giardia-mediated diarrhea remains a major concern, with $>15,000$ cases of Giardia infection reported annually in the USA, accounting for \$34 million in hospital costs [3,4]. Although Giardia infection is potentially treatable, without treatment it can progress to chronic diarrhea, having been implicated in a variety of other gastrointestinal disorders associated with significant morbidity and even mortality. Therefore, it is essential to identify the etiology driving persistent and chronic diarrhea, since although definitive treatment can be lifesaving, its efficacy is limited by the accuracy of available diagnostic tests.

In this issue of Digestive Diseases and Sciences, Jangra et al. endeavored to improve the diagnostic yield of Giardia testing through the implementation of Giardia-specific PCR-based testing of endoscopically obtained duodenal biopsies in patients with persistent and chronic diarrhea [5]. Jangra et al. conducted a prospective cross-sectional study at a single tertiary care center in North India from

\section{Jeffrey A. Berinstein}

jberinst@med.umich.edu

1 Division of Gastroenterology and Hepatology, Department of Internal Medicine, University of Michigan, Ann Arbor, MI, USA

2 Division of Infectious Disease, Department of Internal Medicine, University of Michigan, Ann Arbor, MI, USA
August 2016 to September 2017 on consecutive patients with symptoms of persistent or chronic diarrhea without an identifiable cause. In patients undergoing upper endoscopy as part of their evaluation, PCR testing of duodenal biopsies was compared with stool PCR and stool microscopy. One hundred consecutive subjects (70 adult and 30 pediatric patients) with persistent or chronic diarrhea were evaluated. Of these 100 patients, 30 underwent endoscopic evaluation. Duodenal biopsy PCR detected four additional cases (8\%) of Giardia infection undetected by either stool microscopy or stool PCR. This increased detection rate came at a cost of a high false-negative rate, since duodenal biopsy PCR failed to detect five cases $(10 \%)$ that were positive by stool PCR. The overall diagnostic performance of the duodenal biopsy PCR compared with stool PCR yielded a sensitivity of 54.55\% and specificity of $78.9 \%$. Despite these unexceptional test characteristics, the authors argue that the combination of stool PCR and duodenal biopsy PCR could help increase the detection rate of Giardia infection that may be missed by stool testing alone.

The approach presented by Jangra et al. is novel as it is the first study to evaluate the diagnostic characteristics of duodenal biopsy PCR in a real-world high-prevalence setting [5]. They were able to increase the diagnostic yield of a potentially treatable infection associated with a high mortality and morbidity. This tool could serve as an important adjunctive test for patients at high risk of Giardia-mediated diarrhea despite a negative stool test.

The real-world value of this novel approach remains a major concern-is duodenal biopsy PCR a high-yield diagnostic tool worth implementing? It is unclear whether performing an expensive and invasive endoscopy in a resourcelimited setting where Giardia infection is most relevant is really worth the effort given the small increase in cases detected. Moreover, the lower prevalence of Giardia in a resource-rich country further limits the generalizability of Jangra et al.'s findings. Nevertheless, duodenal biopsy PCR could find its place in the evaluation of chronic functional 
upper gastrointestinal symptoms. Based on emerging literature, Giardia may substantially contribute to the development of idiopathic functional gastrointestinal disorders. Recent publications have revealed that the long-term consequences of Giardia infection include the development of irritable bowel syndrome, functional dyspepsia, and functional bloating [6]. In resource-rich countries, where the prevalence of these conditions is on the rise, upper endoscopy is frequently performed to exclude structural etiologies for these syndromes. In this setting, obtaining duodenal biopsies is frequently performed or could easily be added to standard endoscopy with marginal additional cost and risk. Since the diagnosis of Giardia infection readily suggests a specific treatment approach, increasing the diagnostic yield for this treatable infection would provide both symptomatic relief to the patient and reduce the costs associated with undergoing additional, unnecessary, and expensive testing. It is in this setting that we foresee the greatest value to the authors' approach.

In summary, duodenal biopsy PCR is a welcomed additional tool aimed at increasing the diagnostic yield of Giardia infection testing when performed in combination with or sequentially after stool PCR testing. Not only could this testing strategy identify a treatable cause of persistent and chronic diarrhea in resource-poor settings, however it could also play an important role in the work-up of functional gastrointestinal disorders in resource-rich settings.

\section{Compliance with Ethical Standards}

Conflict of interest JAB reports no relevant disclosures. KR serves as a consultant for Bio-K+ International, Inc.

\section{References}

1. Collaborators GDD. Estimates of global, regional, and national morbidity, mortality, and aetiologies of diarrhoeal diseases: a systematic analysis for the Global Burden of Disease Study 2015. Lancet Infect Dis. 2017;17:909-948.

2. Feng Y, Xiao L. Zoonotic potential and molecular epidemiology of Giardia species and giardiasis. Clin Microbiol Rev. 2011;24:110-140.

3. Scallan E, Hoekstra RM, Angulo FJ, et al. Foodborne illness acquired in the United States-major pathogens. Emerg Infect Dis. 2011;17:7-15.

4. Collier SA, Stockman LJ, Hicks LA, Garrison LE, Zhou FJ, Beach MJ. Direct healthcare costs of selected diseases primarily or partially transmitted by water. Epidemiol Infect. 2012;140:2003-2013.

5. Jangra M, Dutta U, Shah J, et al. Role of polymerase chain reaction in stool and duodenal biopsy for diagnosis of giardiasis in patients with persistent/chronic diarrhea. Dig Dis Sci. (Epub ahead of print). https://doi.org/10.1007/s10620-019-06042-2.

6. Halliez MC, Buret AG. Extra-intestinal and long term consequences of Giardia duodenalis infections. World J Gastroenterol. 2013;19:8974-8985.

Publisher's Note Springer Nature remains neutral with regard to jurisdictional claims in published maps and institutional affiliations. 